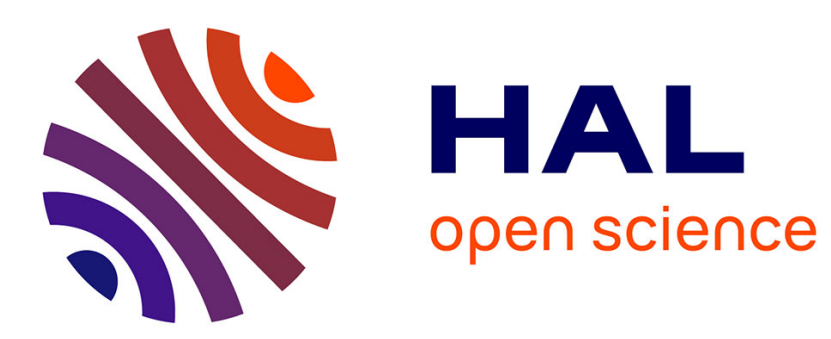

\title{
Suivi de la maturation de couverts de blé par radiométrie dans les domaines visible et proche infra-rouge
}

Frédéric Baret, Gérard Guyot

\section{- To cite this version:}

Frédéric Baret, Gérard Guyot. Suivi de la maturation de couverts de blé par radiométrie dans les domaines visible et proche infra-rouge. Agronomie, 1986, 6 (6), pp.509-516. hal-00884904

\section{HAL Id: hal-00884904 \\ https://hal.science/hal-00884904}

Submitted on 1 Jan 1986

HAL is a multi-disciplinary open access archive for the deposit and dissemination of scientific research documents, whether they are published or not. The documents may come from teaching and research institutions in France or abroad, or from public or private research centers.
L'archive ouverte pluridisciplinaire HAL, est destinée au dépôt et à la diffusion de documents scientifiques de niveau recherche, publiés ou non, émanant des établissements d'enseignement et de recherche français ou étrangers, des laboratoires publics ou privés. 


\title{
Suivi de la maturation de couverts de blé par radiométrie dans les domaines visible et proche infra-rouge
}

Frédéric BARET \& Gérard GUYOT

I.N.R.A., Station de Bioclimatologie, Centre de Recherches d'Avignon, B.P. 9], F 84140 Montfavet

RÉSUMÉ

\begin{abstract}
Les données expérimentales obtenues au cours de 9 années, dans différentes conditions, ont permis d'étudier les relations existant entre les caractéristiques radiométriques de la sénescence de couverts de blé dúrant la phase de maturation et le développement et la croissance des grains.

L'évolution de la sénescence a été suivie en utilisant la différence normalisée (DN), définie à partir des valeurs de réflectance mesurées dans les canaux MSS 5 (rouge) et 7 (proche infrarouge) de Landsat : (DN) $=($ R7 - R5)/ (R7 + R5).

Un modèle simple est proposé. Il permet de déterminer les dates de début et de fin du palier hydrique à partir de la vitesse d'évolution de (DN) en fonction du temps.

Les mesures radiométriques montrent également qu'il est possible d'estimer la production du blé à partir de la vitesse d'évolution de (DN), exprimée en fonction de la somme des températures positives, depuis la floraison. Les relations établies statistiquement sont justifiées par une confrontation avec les travaux récents sur la physiologie de la maturation du blé.
\end{abstract}

Mots clés additionnels : réflectance, indice de végétation, radiométrie, vitesse de sénescence, palier hydrique, rendement.

Additional key words : reflectance, vegetation index, radiometry, senescence rate, water plateau, crop production.

\section{INTRODUCTION}

La phase de maturation des céréales se caractérise par des changements rapides et importants de la structure du couvert (BARET \& HUET, 1979 ; MALET \& BONHOMME, 1981; HUET, 1983) et des propriétés optiques de ses éléments. C'est pourquoi la réflectance du couvert évolue également rapidement dans les domaines du visible et du proche infrarouge, comme le montrent les modèles analytiques de réflectance (VERHOEF \& BUNNIK, 1981) et de nombreuses données expérimentales (KANEMASU, 1974 ; GUYOT \& MALET, 1977 ; TUCKER, 1977 ; LEAMER et al., 1978 ; BAUER et al., 1981).

La phase de maturation est une période critique, particulièrement importante pour les céréales, puisque c'est la période au cours de laquelle se constituent les 
réserves du grain, élément essentiel du rendement final.

Il est donc très utile de situer dans le temps les différentes étapes de cette phase afin de juger de l'adaptation de différents types variétaux ou de techniques culturales aux conditions pédoclimatiques locales.

Les méthodes classiques utilisées pour caractériser l'évolution des grains au cours de cette période sont longues et délicates; c'est pourquoi nous avons mis au point une méthode plus simple et plus rapide, basée sur des mesures radiométriques. Nous avons également examiné dans quelle mesure le suivi radiométrique de la maturation des céréales peut contribuer à une estimation directe du rendement final ou de ses composantes.

\section{MATÉRIEL ET MÉTHODE}

\section{A. Les sites expérimentaux}

Les mesures ont été effectuées de 1976 à 1985 dans 2 sites du Sud-Est de la France : la Station expérimentale de l'I.T.C.F. (Institut Technique des Céréales et Fourrages) à Gréoux-les-Bains et le domaine de l'I.N.R.A. (Institut National de la Recherche Agronomique) à Avignon-Montfavet. Le domaine de l'I.N.R.A. est situé dans la plaine alluviale de la basse vallée du Rhône. Celui de l'I.T.C.F. se trouve dans une petite plaine alluviale de la basse vallée du Verdon à environ $100 \mathrm{~km}$ à l'Est d'Avignon.

Dans ces 2 sites, le climat est de type méditerranéen (GUYOT \& DEVILLARD, 1979) et se caractérise par :

- une très grande variabilité interannuelle de la pluviométrie,

- une période de sécheresse marquée de juin à septembre,

- des températures maximales dépassant souvent $30{ }^{\circ} \mathrm{C}$ au mois de juin, durant la phase de maturation des céréales.

Les sols argilo-limoneux sont peu profonds et reposent sur des lits de galets très filtrants. Leur réserve utile, inférieure à $150 \mathrm{~mm}$, ne permet qu'exceptionnellement une parfaite alimentation hydrique des céréales durant la maturation.

\section{B. Les cultures}

Les données expérimentales utilisées dans cette étude ont été obtenues au cours de 9 campagnes agricoles (de 1976 à 1985) durant lesquelles les conditions climatiques ont présenté une assez large variabilité (années sèches et humides). Comme les mesures ont été effectuées dans les 2 sites expérimentaux, sur diverses variétés de blé, cultivées en sec ou irriguées (tabl. 1), nous avons pu avoir un bon échantillonnage des conditions de développement des plantes dans la région considérée. Nos données ont ainsi été acquises sur des couverts dont la structure géométrique et l'évolution dans le temps ont été très diversifiées.

\section{Les mesures biologiques}

Sur chacune des parcelles étudiées, 2 types de mesures ont été effectués :

\section{Suivi précis de la phase de maturation}

La phase de maturation des céréales est la période au cours de laquelle se constituent les réserves des grains. Depuis les travaux de GESLIN \& JONARD (1948) et JONARD (1960), elle est classiquement divisée en 3 périodes à partir de la fécondation (fig. 1) :

- formation des enveloppes et multiplication des cellules de l'endosperme (durée 20 à $25 \mathrm{j}$ ),

- palier hydrique, durant lequel le poids d'eau du grain est constant (durée 10 à $20 \mathrm{j}$ ),

- dessèchement du grain (durée 15 à $20 \mathrm{j}$ ).

Les mesures entreprises sont destinées à suivre l'évolution des teneurs en eau et en matière sèche des grains, de manière à déterminer le début et la fin du palier hydrique. Conformément à la méthodologie proposée par MALET (1979) et MALET \& GURNADE (1981) et aux résultats expérimentaux de BARET \& HUET (1979), un échantillon de 10 épis entiers, non égrenés, est prélevé au hasard, 2 à 3 fois par semaine, sur la strate dominante de chaque parcelle. Sur chaque épi un lot de 10 grains est prélevé à partir du $4^{\text {e }}$ étage d'épillets, qui correspond au même segment d'organogenèse de l'épi (VINCENT \& MALET, 1977). Nous avons mesuré leurs poids frais et $\sec (24 \mathrm{~h}$ à l'étuve à $105^{\circ} \mathrm{C}$ ).

Pour plus de commodité et de précision, les dates de début et de fin de palier hydrique sont déterminées comme les dates auxquelles les teneurs en eau des grains atteignent respectivement 66 et 44 p. 100 (BALLOT, 1962 ; MIGUET, 1983).

\section{Estimation du rendement et de ses composantes}

A la maturité, le rendement ainsi que ses principales composantes (nombre d'épis par $\mathrm{m}^{2}$, nombre de grains par épi, poids de 1000 grains), sont estimés à partir de 3 prélèvements de 2 rangs adjacents, sur $1 \mathrm{~m}$ de long (correspondant à une surface totale récoltée de $1,05 \mathrm{~m}^{2}$ pour un espacement des rangs de $0,175 \mathrm{~m}$ ).

\section{Mesures radiométriques}

La réflectance des différentes parcelles étudiées est mesurée 2 à 3 fois par semaine, par temps clair, au midi solaire, dans les 4 bandes spectrales des satellites Landsat (R4 : 500-600 nm, R5 : 600-700 nm, R6 : $700-800 \mathrm{~nm}, \mathrm{R} 7: 800-1100 \mathrm{~nm}$ ). Pour cela, nous avons utilisé 2 radiomètres (Exotech $100 \mathrm{~A}$ ) reliés à une centrale d'acquisition de données et mesurant simultanément l'éclairement et la luminance du couvert. Le radiomètre mesurant la luminance est fixé à l'extrémité d'une potence et placé à plus de $1,5 \mathrm{~m}$ audessus du couvert qu'il vise verticalement. Il est équipé d'objectifs ayant une ouverture de $15^{\circ}$. Le $2^{\mathrm{e}}$ radiomètre mesure l'éclairement et vise le ciel verticalement. Il est équipé d'objectifs diffusants ayant une ouverture de $180^{\circ}$.

Sur chacune des parcelles étudiées les mesures sont répétées au moins 9 fois, de manière à avoir une précision satisfaisante sur les valeurs moyennes. 
TABLEAU 1

Ensemble des données utilisées. La colonne « culture » indique si les plantes ont été irriguées (I) ou conduites en sec (S). $P M G$ donne le poids de 1000 grains. Les autres expressions sont définies dans le texte.

Experimental data used in this study. "Variété ": wheat cultivar. "Culture » : (I) irrigated, (S) without irrigation. "Prod. P 》 : crop yield expressed in $q / h a(10 q=1 \mathrm{l})$. « Nombre grains par $\mathrm{m}^{2}$ " Kernel number per unit area. "PMG» 1000 Kernel weight. The other expressions are defined in the text.

\begin{tabular}{|c|c|c|c|c|c|c|c|c|c|c|c|}
\hline Année & Lieu & Variété & $\begin{array}{l}\text { Culture } \\
\text { I }\end{array}$ & & $\begin{array}{l}\text { Prod. P } \\
(\mathrm{q} / \mathrm{ha})\end{array}$ & $\begin{array}{c}\text { Nombre } \\
\text { grains } \\
\text { par } \mathrm{m}^{2}\end{array}$ & $\begin{array}{l}\text { P.M.G. } \\
\text { g }\end{array}$ & $\frac{\mathrm{d}(\mathrm{N})}{\mathrm{dt}}$ & $\begin{array}{c}\frac{\mathrm{d}(\mathrm{DN})}{\mathrm{d}\left(\sum \mathrm{T}\right)} \\
10^{-4} \\
\mathrm{~J}^{-1} \cdot{ }^{\circ} \mathrm{C}^{-1}\end{array}$ & $d-\mathbf{J}$ & $f-a$ \\
\hline 1976 & Gréoux & $\begin{array}{c}\text { Talent } \\
\text { Capitole }\end{array}$ & & $\begin{array}{l}* \\
*\end{array}$ & 53,3 & 22400 & 23,8 & $\begin{array}{l}-0,026 \\
-0,031\end{array}$ & $-11,59$ & $\begin{array}{l}-0,7 \\
-3,5\end{array}$ & $\begin{array}{l}16,3 \\
11,3\end{array}$ \\
\hline 1977 & Gréoux & $\begin{array}{c}\text { Talent } \\
\text { Talent } \\
\text { Courtot }\end{array}$ & $\begin{array}{l}* \\
*\end{array}$ & * & $\begin{array}{l}47,0 \\
43,6\end{array}$ & $\begin{array}{l}14500 \\
13090\end{array}$ & $\begin{array}{l}32,4 \\
33,3\end{array}$ & $\begin{array}{l}-0,017 \\
-0,017 \\
-0,014\end{array}$ & $\begin{array}{l}-9,95 \\
-\quad 9,08\end{array}$ & $\begin{array}{r}+7,0 \\
5,7 \\
12,8\end{array}$ & $\begin{array}{l}27,0 \\
22,7 \\
33,8\end{array}$ \\
\hline 1978 & Avignon & $\begin{array}{l}\text { Talent } \\
\text { Talent }\end{array}$ & & * & & & & $\begin{array}{r}-0,017 \\
-0,018\end{array}$ & & $\begin{array}{l}9,7 \\
8,1\end{array}$ & $\begin{array}{l}24,7 \\
23,1\end{array}$ \\
\hline 1979 & Avignon & $\begin{array}{c}\text { Talent } \\
\text { Capitole }\end{array}$ & * & & 47,7 & 14820 & 32,2 & $\begin{array}{l}-0,033 \\
-0,033\end{array}$ & $-14,41$ & $\begin{array}{l}-3,0 \\
-0,9\end{array}$ & $\begin{array}{l}15,1 \\
16,3\end{array}$ \\
\hline 1980 & Avignon & Talent & & $*$ & & & & $-0,022$ & & 3,0 & 24,0 \\
\hline 1983 & Gréoux & $\begin{array}{c}\text { Talent } \\
\text { Capitole } \\
\text { Castan } \\
\text { Fidel }\end{array}$ & & $\begin{array}{l}* \\
* \\
* \\
*\end{array}$ & 53,0 & 19209 & 27,6 & $\begin{array}{r}-0,022 \\
-0,021 \\
-0,028 \\
-0,026\end{array}$ & $-9,10$ & $\begin{array}{l}3,5 \\
2,1 \\
3,0 \\
1,0\end{array}$ & $\begin{array}{l}22,5 \\
20,1 \\
20,1 \\
20,1\end{array}$ \\
\hline 1984 & Avignon & Talent & & * & 56,1 & & & $-0,026$ & $-11,61$ & 2,2 & 14,2 \\
\hline 1984 & Gréoux & $\begin{array}{l}\text { Talent } \\
\text { Talent } \\
\text { Talent } \\
\text { Talent }\end{array}$ & * & * & $\begin{array}{l}74,3 \\
69,3 \\
62,1 \\
62,3\end{array}$ & $\begin{array}{l}22990 \\
20620 \\
21490 \\
19970\end{array}$ & $\begin{array}{l}32,3 \\
33,6 \\
28,9 \\
31,2\end{array}$ & $\begin{array}{l}-0,031 \\
-0,041 \\
-0,028 \\
-0,032\end{array}$ & $\begin{array}{l}-20,06 \\
-23,81 \\
-19,79 \\
-19,05\end{array}$ & $\begin{array}{r}-2,8 \\
-2,0 \\
0,4 \\
1,6\end{array}$ & $\begin{array}{l}12,6 \\
11,5 \\
12,9 \\
13,8\end{array}$ \\
\hline 1985 & Avignon & $\begin{array}{c}\text { Talent } \\
\text { Arcane } \\
\text { Arminda }\end{array}$ & & $\begin{array}{l}* \\
* \\
*\end{array}$ & 60,7 & 19970 & 30,4 & $\begin{array}{l}-0,033 \\
-0,026 \\
-0,014\end{array}$ & $-11,70$ & $\begin{array}{r}-1,0 \\
3,0 \\
10,1\end{array}$ & $\begin{array}{l}14,8 \\
19,7 \\
28,6\end{array}$ \\
\hline
\end{tabular}

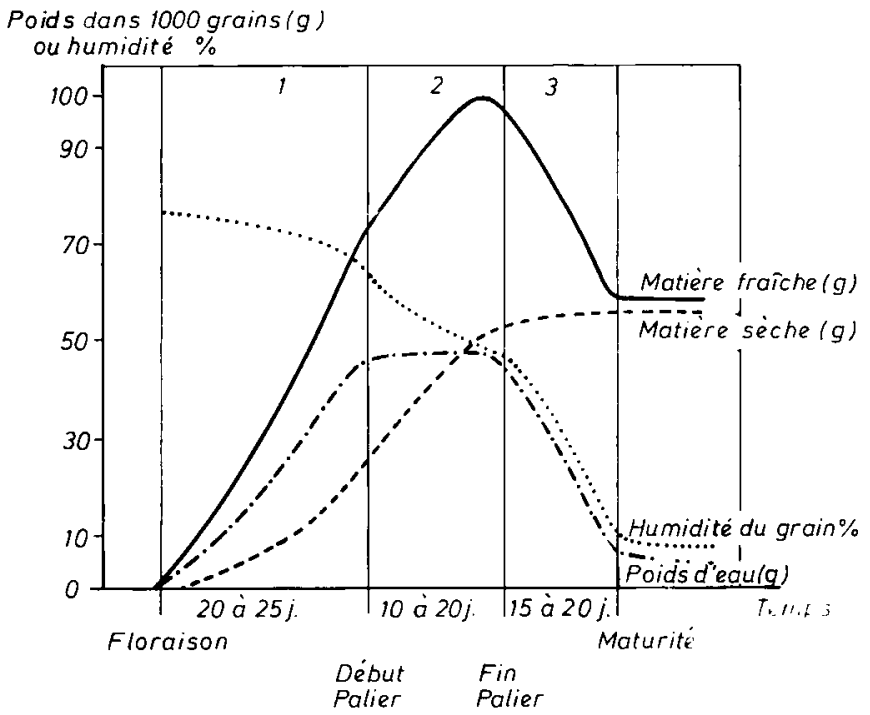

Figure 1

Evolution du poids de matière sèche, de matière fraîche et d'eau des grains ainsi que du pourcentage d'eau qu'ils contiennent, durant la phase de maturation (d'après GESLIN \& JONARD, 1948).

Dry matter, fresh matter and water in grain, and percent water content, during the ripening period (after GESLIN \& JONARD, 1948).

\section{RÉSULTATS EXPÉRIMENTAUX ET DISCUSSION}

\section{A. Caractérisation radiométrique de l'évolution de la végétation}

\section{Choix d'un indice de végétation}

Durant la phase de maturation d'une culture de blé, on observe une diminution rapide de la réflectance dans le proche infrarouge et une augmentation plus lente dans le visible, comme le montrent de nombreux résultats expérimentaux (GUYOT \& MALET, 1977; TUCKER, 1977 ; LEAMER et al., 1978 ; BAUER et al., 1981 ; AHLRICHS \& BAUER, 1983 ; GUYOT, 1984).

Pour caractériser l'évolution d'un couvert végétal et limiter l'influence de facteurs externes (nébulosité, hauteur du soleil...) ou internes (propriétés optiques du sol), de nombreux auteurs ont proposé de combiner des données de réflectance obtenues dans différentes bandes de longueur d'onde, afin d'obtenir des indices de végétation.

PERRY \& LAUTENSCHLAGER (1984) ont montré qu'un bon nombre d'indices couramment utilisés sont 
fonctionnellement équivalents. Aussi, en nous appuyant sur les travaux de AASE \& SIDDOWAY (1980, 1981), AASE et al. (1984), TUCKER et al. (1981), MARKHAM et al. (1981), WIEGAND \& RICHARDSON (1984), avons nous choisi la différence normalisée (DN) :

$$
(\mathrm{DN})=(\mathrm{R} 7-\mathrm{R} 5) /(\mathrm{R} 7+\mathrm{R} 5) .
$$

Cet indice est très étroitement lié à l'indice foliaire vert ou encore à la partie photosynthétiquement active du rayonnement intercepté par la culture.

\section{Evolution de l'indice de végétation (DN)}

La figure 2 représente l'évolution type de (DN) au cours du cycle végétatif de céréales. Lorsque la végétation s'installe sur le sol, l'indice de végétation (DN) croît régulièrement. En effet la réflectance des plantes dans le proche infrarouge (R7) est généralement plus élevée que celle du sol alors que, dans le domaine du visible (R5), elle lui est inférieure (tabl. 2). A partir du gonflement, la couverture du sol est pratiquement totale et l'indice (DN) atteint un palier qui se maintient jusqu'à la floraison. Au-delà, durant la phase de maturation, la décroissance de la réflectance dans le proche infrarouge et son augmentation dans le visible entraînent une diminution de (DN).

Pour décrire l'évolution de (DN), durant la phase de maturation, nous avons choisi un modèle très simple à 3 paramètres (fig. 3) :

- $\mathrm{h}$ : hauteur moyenne du palier de (DN),

- d(DN)/dt : vitesse de décroissance de (DN). Elle est estimée par la pente de la droite de régression, ajustée aux points expérimentaux obtenus durant la phase de décroissance de $(\mathrm{DN})(0,75>(\mathrm{DN})>0,43)$. Les ajustements linéaires effectués ont des coefficients de corrélation supérieurs à 0,95 ,

- a : date correspondant au point d'intersection du palier de (DN) avec la droite ajustée à la phase de décroissance.
TABLEAU 2

Réflectances moyennes de feuilles de blé et de sol nu et sec dans les canaux MSS 5 (rouge) et 7 (proche infrarouge) de Landsat (d'après BARET \& HUET, 1979).

Mean reflectance of wheat leaves and bare and dry soil in the MSS 5 (red) and 7 (near infrared) channels of Landsat (after BARET \& HUET, 1979).

\begin{tabular}{lcc}
\hline \multicolumn{1}{c}{ Domaine spectral } & MSS 5 & MSS7 \\
& $600-700 \mathrm{~nm}$ & $800-1100 \mathrm{~nm}$ \\
\hline Feuille adulte verte & $3 \%$ & $45 \%$ \\
Feuille sénescente & $40 \%$ & $57 \%$ \\
Sol nu ét sec & $22 \%$ & $26 \%$ \\
\hline
\end{tabular}

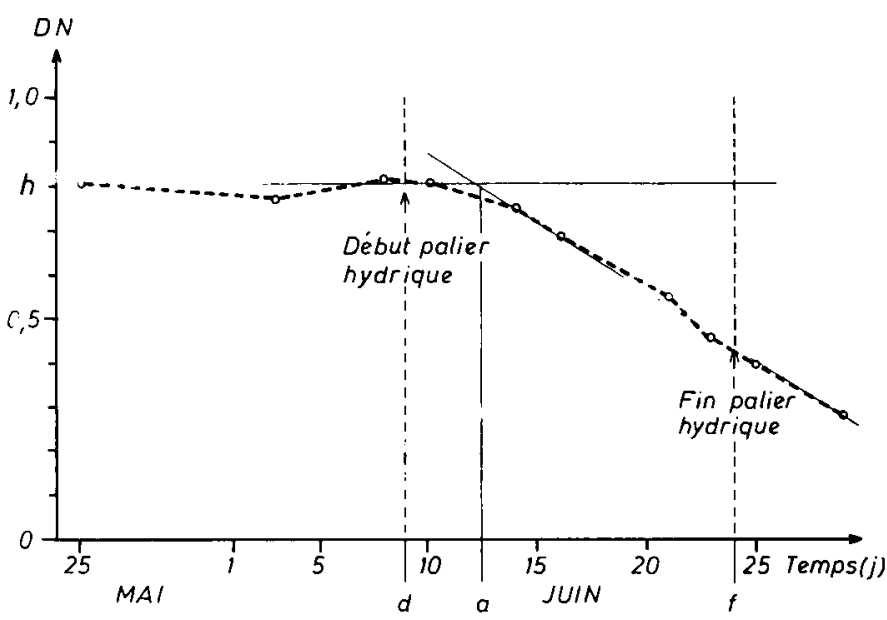

Figure 3

Evolution de (DN) au cours de la maturation du blé et définition des dates caractéristiques utilisées dans le texte:

Change in (DN) during ripening of wheat and definition of characteristic dates used in the text :

$a:$ début de la décroissance de (DN); beginning of (DN) decrease. $d$ : début du palier hydrique; beginning of the water plateau. $f:$ fin du palier hydrique; end of the water plateau.

$h$ : hauteur du plateau de saturation de (DN) ; level of (DN) saturation plateau.

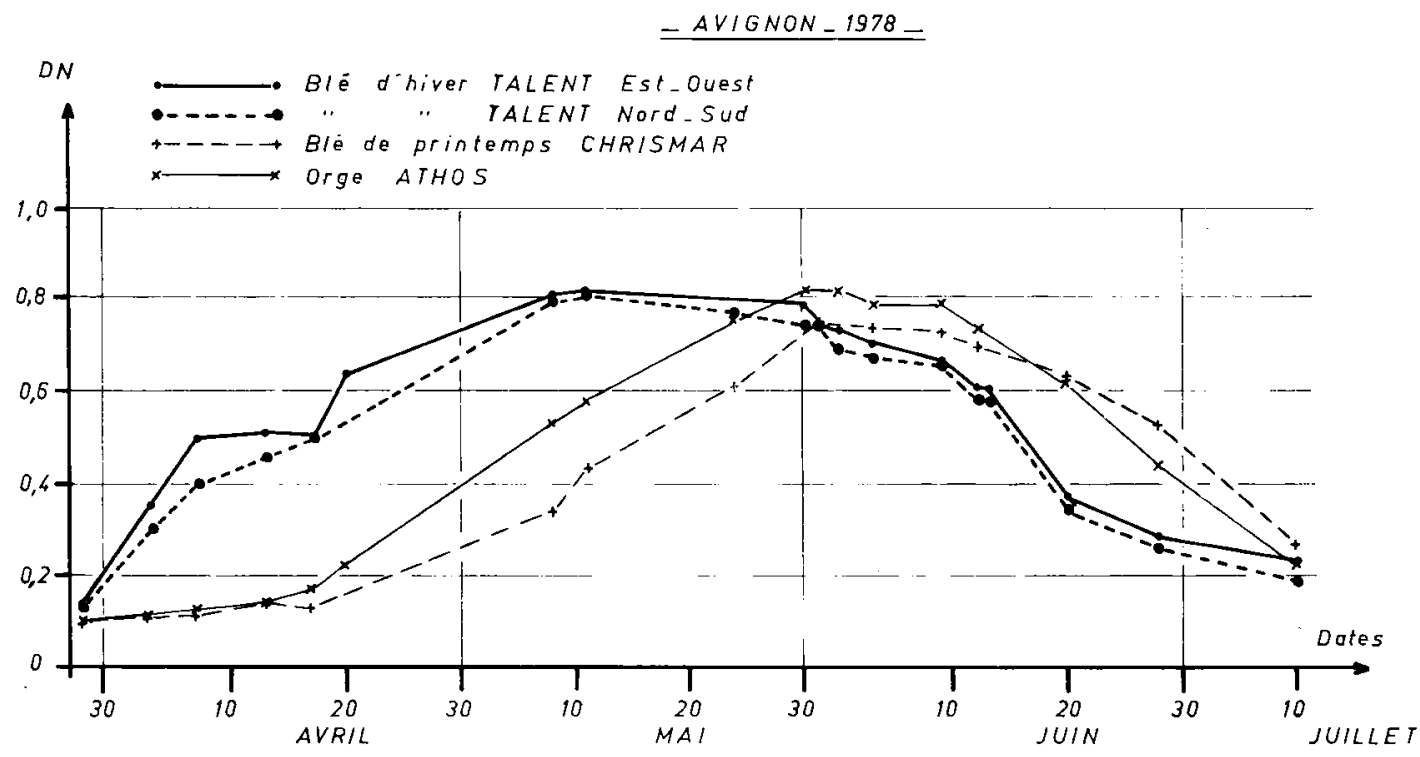

Figure 2

Evolution au cours du temps de la différence normalisée (DN) pour différents couverts de céréales (Avignon, 1978).
Progress with time of the normalized difference for different cereal canopies (Avignon, 1978). 
3. Application des résultats des mesures radiométriques au suivi de la maturation

L'examen des figures 2 et 3 laisse penser que la décroissance de (DN) doit être liée au palier hydrique, comme l'ont fait remarquer GUYOT \& MALET (1977). Cette hypothèse a été testée sur l'ensemble des données dont nous disposions et nous avons constaté que l'intervalle de temps, séparant la date de début (d) du palier hydrique de la date (a) du début de la phase de décroissance, varie de -4 à $+13 \mathrm{j}$ (tabl. 1) et qu'il est étroitement corrélé à la vitesse de décroissance de (DN) : d(DN)/dt (fig. 4). Nous avons ainsi obtenu :

$$
\text { (d) } \begin{aligned}
-(\mathrm{a}) & =555 \cdot \mathrm{d}(\mathrm{DN}) / \mathrm{dt}+16,7 \\
(\mathrm{n} & =23, \mathrm{r}=0,89) .
\end{aligned}
$$

Cette relation montre ainsi la possibilité de déterminer la date (d) de début du palier hydrique à partir de mesures radiométriques.

En utilisant la même démarche appliquée à la détermination de la date (f) de la fin du palier hydrique nous avons obtenu la relation :

$$
\text { (f) } \begin{aligned}
-(\mathrm{a}) & =738 \cdot \mathrm{d}(\mathrm{DN}) / \mathrm{dt}+38,0 \\
(\mathrm{n}= & =23, \mathrm{r}=0,89) .
\end{aligned}
$$

Les relations (1) et (2) indiquent que la durée du palier hydrique (f) - (a) devrait être liée linéairement à la vitesse de décroissance de (DN) en fonction du temps. Le coefficient de corrélation entre la durée
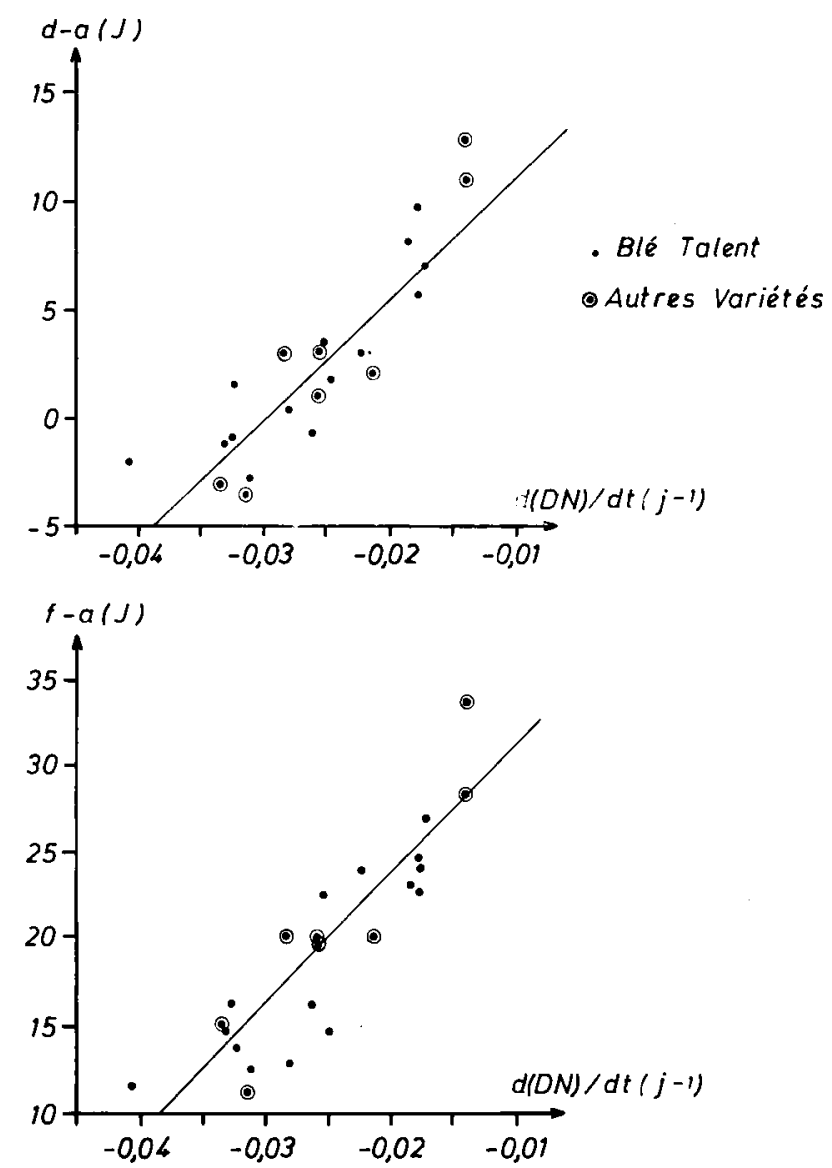

Figure 4

Relations entre $(d-a)$ et $(f-a)$ et la vitesse d'évolution de $(D N)$ en fonction du temps.

Relationships between $(d-a)$ and $(f-a)$ and the rate of change of (DN) with time. du palier hydrique et $d(D N) / d t$ est en effet très significatif bien que faible $(r=0,55, n=23)$. Cette relative dispersion de nos données est peut-être simplement due à l'incertitude avec laquelle la durée du palier hydrique est connue. En effet, les dates de début (d) et de fin (f) sont déterminées à 1 à $2 \mathrm{j}$ près et comme dans nos essais les durées de palier hydrique ont varié entre 12 et $21 \mathrm{j}$, l'erreur relative sur ces déterminations a pu atteindre 33 p. 100 . Quoi qu'il en soit, nos résultats montrent bien qu'aux paliers hydriques les plus longs correspondent les vitesses d'évolution de (DN) les plus faibles (sénescence plus lente du couvert).

\section{B. Estimation des rendements à partir des mesures radiométriques}

Comme il semblait possible de déterminer la chronologie de la maturation à partir de mesures radiométriques, nous avons alors pensé que l'on devrait également pouvoir relier la vitesse d'évolution de (DN) à la production finale. Nous avons ainsi déterminé la corrélation existant entre la production $P(q / h a)$ et $\mathrm{d}(\mathrm{DN}) / \mathrm{dt}$ pour la variété « Talent » :

$$
\begin{gathered}
\mathrm{P}=-936 \cdot \mathrm{d}(\mathrm{DN}) / \mathrm{dt}+31,2 \\
(\mathrm{n}=11, \mathrm{r}=0,713) .
\end{gathered}
$$

Cette liaison significative est à rapprocher des résultats obtenus par IDSO et al., 1980. Ces auteurs ont en effet établi le même type de relation entre la vitesse de sénescence, mesurée avec un radiomètre, et la production finale de grains. Cependant, pour caractériser l'état de la végétation, ils ont utilisé l'indice de végétation transformé (TVI6) au lieu de la différence normalisée (DN). Selon TUCKER et al. (1981), l'indice (TVI6) qui s'écrit :

$$
(\mathrm{TVI})=\sqrt{\frac{\mathrm{R} 6-\mathrm{R} 5}{\mathrm{R} 6+\mathrm{R} 5}+0,5}
$$

permet de mieux séparer le sol de la végétation dans certaines conditions. Ils ont ainsi établi la relation empirique donnant la production $\mathrm{P}(\mathrm{q} / \mathrm{ha})$ de la variété de blé dur "Produra » sous le climat aride de l'Arizona :

$\mathrm{P}=-4136 . \mathrm{d}(\mathrm{TVI}$ ) $/ \mathrm{dt}-20,8$.

Comme nous ne disposions pas dans nos archives des données nous permettant de calculer (TVI6) pour tous les essais, nous avons essayé d'établir une relation entre (DN) et (TVI6) dans nos conditions expérimentales, car nous avons pu montrer l'existence d'une liaison étroite entre R6 et R7. Pour cela nous avons calculé les 2 indices pour une série de 182 mesures effectuées en 1978 et 1979 durant la phase de maturation de céréales. Nous avons ainsi obtenu la relation :

$$
\begin{gathered}
(\mathrm{DN})=1,922 .(\mathrm{TVI} 6)-1,330 \\
(\mathrm{n}=182, \mathrm{r}=0,998) .
\end{gathered}
$$

Les informations obtenues en utilisant (DN) ou (TVI6) sont donc équivalentes. Si nous transformons la relation (3) en utilisant la relation (5) nous obtenons la relation (6) qui donne la production du blé "Talent » en fonction de (TVI6) :

$\mathrm{P}=-1799 . \mathrm{d}($ TVI6) $/ \mathrm{dt}+31,2$. 


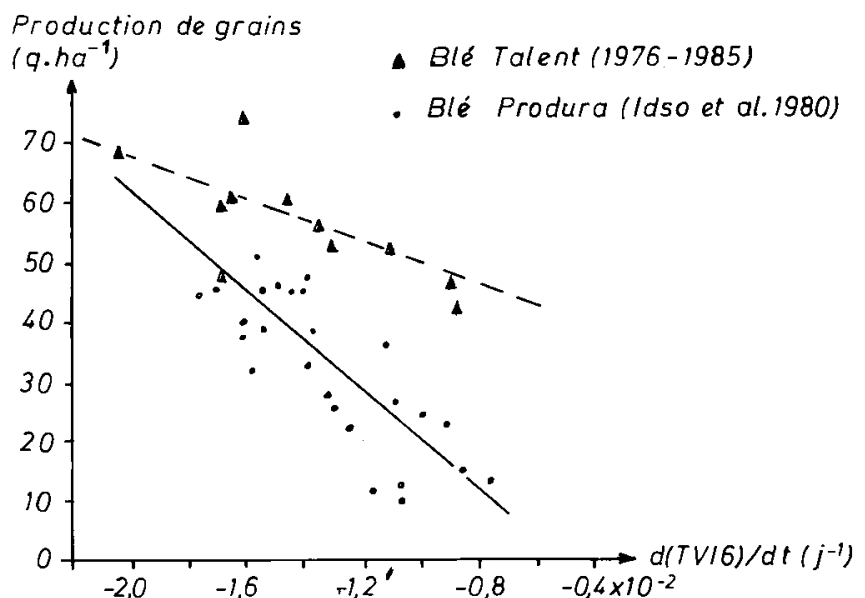

Figure 5

Relation entre la production du blé et la vitesse d'évolution de (TVI6). Les mesures effectuées en France sont comparées aux résultats de IDSo et al. (1980).

Relationship between wheat production and change in (TVI6). Data collected in France compared with results of IDSO et al. (1980).

Nous pouvons remarquer sur la figure 5 que, malgré d'importantes différences de conditions expérimentales (matériel végétal, climat, techniques culturales, méthode de mesure radiométrique), les relations (4) et (6) décrivent bien le même phénomène biologique et les points correspondant aux 2 séries de données sont en bon accord. Ainsi, la production de grain est d'autant plus élevée que la décroissance de (DN) ou (TVI6), en fonction du temps, est plus rapide.

Comme tout processus de croissance ou de développement dépend de la température, nous avons remplacé l'échelle des temps par une échelle proportionnelle à la somme des températures positives (DURAND, 1969) depuis la floraison : $\Sigma \mathrm{T}$. La vitesse d'évolution de $(\mathrm{DN})$ s'écrit alors : $\mathrm{d}(\mathrm{DN}) / \mathrm{d} \hat{k} \Sigma \mathrm{T})$. En prenant en compte la température (fig. 6), on obtient alors la relation (7) donnant la production $\mathrm{P}(\mathrm{q} / \mathrm{ha})$ :

$$
\begin{gathered}
\mathrm{P}=-1,50 \cdot 10^{4} \cdot \mathrm{d}(\mathrm{DN}) / \mathrm{d}(\Sigma \mathrm{T})+35,4 \\
(\mathrm{n}=11, \mathrm{r}=-0,82) .
\end{gathered}
$$

La légère augmentation du coefficient de corrélation de la relation (7) par rapport à celui de la relation (3), bien que non significative, est confortée par les travaux de GESLIN \& JONARD (1948) MASSÉ (1981) et TRIBOİ et al. (1985) montrant que la dynamique de la maturation du grain dépend des conditions de température durant cette période.

Une analyse des processus intervenant durant la maturation permet de mieux comprendre la signification biologique de la relation (7). TRIBOÏ et al. (1985) montrent que le poids d'un grain peut être considéré comme le produit de 2 facteurs :

- La vitesse de remplissage du grain qui est principalement déterminée par la quantité d'assimilats accumulés avant la floraison. Ce stock d'assimilats est positivement corrélé au nombre de grains par unité de surface,

- La durée de remplissage du grain qui est inversement corrélée à la vitesse de remplissage du grain ; mais un allongement de la durée du remplissage du grain ne compensera jamais entièrement une faible vitesse de remplissage.
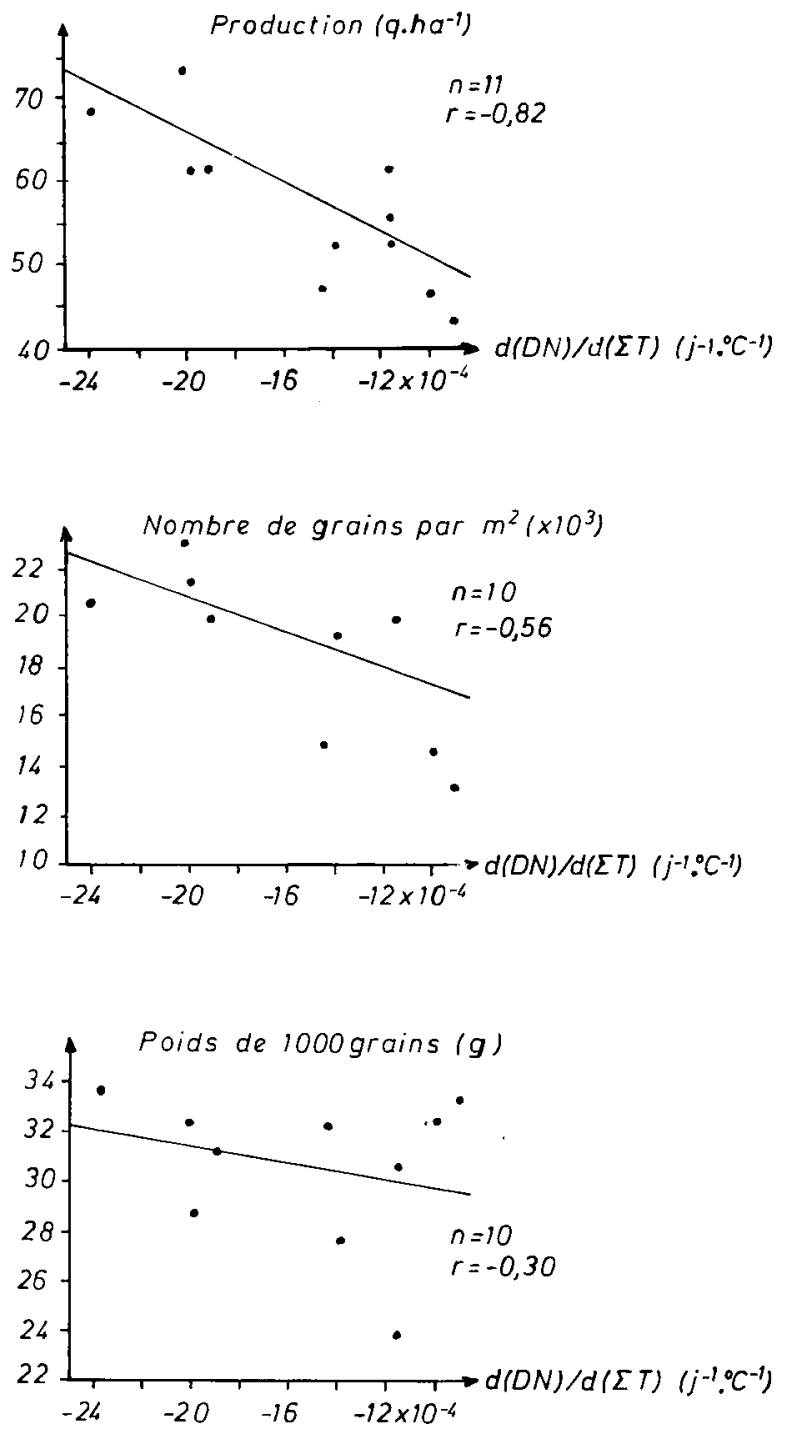

Figure 6

Relations entre le rendement du blé «Talent ", le nombre de grains par unité de surface, le poids de l 000 grains et la vitesse d'évolution de (DN), exprimées en fonction de la somme des températures positives, comptées depuis la floraison.

Relationships, for wheat c $v$. 'Talent', between yield, number of kernels per unit area, 1 000-kernel weight and rate of change of $(D N)$, expressed as a sum of positive temperatures calculated from flowering.

Ainsi, une forte production, résultat du produit d'un grand nombre de grains par unité de surface par un poids de 1000 grains important, correspondra à une vitesse de remplissage du grain élevée et donc à une maturation rapide. C'est bien ce qu'exprime la relation statistique (7) entre production et vitesse d'évolution de (DN).

La vitesse d'évolution de (DN) semble donc être surtout liée au nombre $\mathrm{N}$ de grains par unité de surface (composante du rendement qui reflète l'état de la culture à la floraison) et beaucoup moins au poids de 1000 grains (PMG). C'est ce que montrent les relations (8) et (9) (fig. 6) :

$$
\begin{gathered}
\mathrm{N}=-3,62 \cdot 10^{6} \cdot \mathrm{d}(\mathrm{DN}) / \mathrm{d}(\Sigma \mathrm{T})+11972 \\
(\mathrm{n}=10, \mathrm{r}=0,56) . \\
\mathrm{PMG}=-\begin{array}{c}
1,72 \cdot 10^{3} \cdot \mathrm{d}(\mathrm{DN}) / \mathrm{d}(\Sigma \mathrm{T})+28,02 \\
(\mathrm{n}=10, \mathrm{r}=0,30) .
\end{array}
\end{gathered}
$$




\section{CONCLUSION}

Les résultats expérimentaux présentés montrent qu'il est possible de suivre la chronologie de la maturation du blé à partir de mesures radiométriques. La différence normalisée (DN) permet de suivre la sénescence d'un couvert et nous avons montré que l'on peut ainsi déterminer les dates de début et de fin de palier hydrique, pour les différentes variétés utilisées dans les essais, avec une précision comparable à celle de la méthode classique. L'application de cette technique pourrait permettre de simplifier les procédures expérimentales qui sont utilisées pour caractériser l'adaptation de types variétaux ou de techniques culturales à des conditions pédoclimatiques données.

Le suivi radiométrique de la sénescence de couverts de blé semble offrir une possibilité d'estimer la production finale de grain. Cette estimation sera améliorée si on exprime la vitesse d'évolution de (DN) non pas simplement en fonction du temps, mais en fonction de la somme des températures positives, calculée depuis la floraison.

La relation statistique, établie expérimentalement, entre la vitesse de sénescence du couvert et sa production, semble reposer sur des relations fonctionnelles entre la vitesse de remplissage des grains et la vitesse de sénescence du couvert. Comme la vitesse de remplissage des grains est, en grande partie, déterminée à la floraison, le suivi radiométrique de la maturation de couverts de blé sera donc surtout révélateur de leur état à la floraison. Par contre, la vitesse de sénescence du couvert ne semble paradoxalement que peu révélatrice des variations du poids de 1000 grains dues aux facteurs pédoclimatiques intervenant durant la maturation.

Les relations qui ont été discutées reposent sur un nombre limité de mesures effectuées uniquement sur la variété de blé «Talent». Pour vérifier leur domaine de validité et accroître leur précision, il sera nécessaire non seulement de multiplier et de diversifier les conditions expérimentales, mais également d'affiner les observations afin d'essayer d'en comprendre le déterminisme.

Ces résultats montrent l'intérêt d'un suivi serré de l'évolution radiométrique des couverts végétaux. Cela nécessitera donc d'avoir, pour les applications pratiques, des fréquences d'observation beaucoup plus élevées que ce que permettent les satellites d'observation de la terre actuels.

Recu le 17 septembre 1984. Accepté le 3 février 1986.

\section{REMERCIEMENTS}

Les auteurs tiennent à remercier l'I.T.C.F. qui a donné toutes les facilités pour que les différentes expériences puissent avoir lieu sur son domaine expérimental de Gréoux-les-Bains.

\section{RÉFÉRENCES BIBLIOGRAPHIQUES}

Aase J. K., Siddoway F. H., 1980. Determining winter wheat stand densities using spectral reflectance measurements. Agron. J., 72, 149-152.

Aase J. K., Siddoway F. H., 1981. Assessing winter wheat dry matter production via spectral reflectance measurements. Rem. Sensing Environ., 11, 267-277.

Aase J. K., Siddoway F. H., Millard J. P., 1984. Spring wheat leaf phytomass and yield estimates from airborne scanner and hand-held radiometer measurements. Int. J. Rem. Sensing, 5 (5), 771-781.

Ahlrichs J. S., Bauer M. E., 1983. Relation of agronomic and multispectral reflectance characteristics of spring wheat canopies. Agron. J., 75, 987-993.

Ballot X., 1962. Contribution à l'étude de la maturation du grain chez différents génotypes de Triticum en fonction du climat et des méthodes culturales. Mém. Conservatoire National des Arts et Métiers, Paris, 79 p.

Baret F., Huet M., 1979. Contribution à l'utilisation agronomique de la télédétection: analyse des relations entre la structure d'un couvert végétal, son état physiologique et les données radiométriques. Rapport de fin de stage, I.N.R.A., Station de Bioclimatologie, 84140 Montfavet (France), 115 p.

Bauer M. E., Daughtry C. S. T., Vanderbilt V. C., 1981. Spectral agronomic relationships of maize, soybean and wheat canopies. In $C$. R. Coll. Int. sur les Signatures Spectrales d'Objets en Télédétection, Avignon, septembre 1981. Les Colloques de l'I.N.R.A. $N^{\circ} 5,261-272$.

Durand R., 1969. Signification et portée des sommes de températures. Bull. tech. Inf. Min. Agric., 238, 185-190.

Geslin H., Jonard P., 1948. Maturation du blé et climat. Ann. Nutr. Aliment., 2, 361-371.

Guyot G., 1984. Caractérisation spectrale des couverts végétaux, application à la télédétection. Bull. Soc. Fr. Photogram. Télédétect., 95 (3), 5-22.
Guyot G., Devillard R., 1979. Le climat d'Avignon, I.N.R.A., Station de Bioclimatologie, 84140 Montfavet (France), 43 p.

Guyot G., Malet P., 1977. Etude de l'évolution de la réflectance d'un blé d'hiver dans les 4 bandes MSS de Landsat, In E. A. Godby \& I. Otterman : «The contribution of space observation to global food information systems ». COSPAR. Adv. in Space Explor., Vol. 2, Pergamon Press., 51-55.

Huet M., 1983. Evolution des paramètres de structure et de biomasse d'un couvert de blé. Utilisation des techniques de télédétection micro-ondes. Thèse Doct. Ing., Univ. Paul Sabatier, Toulouse (Science), N $853,102 \mathrm{p}$.

Idso S. B., Pinter P. J., Jackson R. D., Reginato R. J., 1980. Estimation of grain yield by crop senescence rates. Rem. Sensing Environ., 7, 273-276.

Jonard P., 1960. Etude des caractéristiques physiques du grain de blé. Ann. Amélior. Plantes, 10 (3), 237-273.

Kanemasu E. T., 1974. Seasonal canopy reflectance patterns of wheat, sorghum and soybean. Rem. Sensing Environ., 3, 43-47.

Leamer R. W., Noriega J. R., Wiegand C. L., 1978. Seasonal changes in reflectance of two wheat cultivars. Agron. J., 70, 113-118.

Malet P., 1979. Liaison statistique entre le développement et la croissance des plantes : modèle simplifié pour l'analyse de la croissance des organes. Ann. Agron., 30 (5), 415-450.

Malet P., Bonhomme R., 1981. Signification biologique de la structure d'un couvert de blé ou de maîs, conséquences pour l'interprétation des données de télédétection. In $C$. $R$. Colloq. Int, sur les Signatures Spectrales d'Objets en Télédétection, Avignon, septembre 1981. Les Colloques de l'I.N.R.A. $\mathrm{N}^{\circ}$ 5, 239-250.

Malet P., Gurnade J. C., 1981. Macrophysiologie de la maturation du blé en conditions naturelles. I. Notion de représentativité chronologique et normes d'échantillonnage. Agronomie, 1 (3), 235-242.

Markham B. L., Kimes D. S., Tucker C. J., 1981. Temporal spectral response of corn canopy. Photogram. Eng. Rem. Sensing, 48 (11), 1599-1605 
Massé J., 1981. La maturation du blé dépend surtout du climat. Perspect. Agric., 51, 13-22.

Miguet J. N., 1983. Etude de différentes méthodes de suivi de la phase de maturation du blé d'hiver. Notion de représentativité quantitative. Mém. fin d'études, E.N.S.A. de Rennes, 93 p.

Perry C. R., Lautenschlager L. F., 1984. Functional equivalence of spectral vegetation indices. Rem. Sensing Environ., 14, 169-182.

Triboï R., Rousset M., Lemercier E., 1985. Etude de l'interaction génotype-milieu chez le blé d'hiver. Elaboration du poids du grain. I.N.R.A., ATP «Ecophysiologie du blé ». C. R. intermédiaire, campagne 1984, juillet $1985,67-104$.

Tucker C. J., 1977. Spectral estimation of grass canopy variables. Rem. Sensing Environ., 6, 11-26.
Tucker C. J., Holben B. N., Elgin J. H., Me Murtey J. E., 1981. Remote sensing of total dry matter accumulation in winter wheat. Rem. Sensing Environ., 11, 171-189.

Verhoef W., Bunnik N. J. J., 1981. Influence of crop geometry on multispectral reflectance determined by the use of canopy reflectance models. In C. R. Coll. Int. sur les Signatures Spectrales d'Objets en Télédétection, Avignon, septembre 1981. Les Colloques de l'I.N.R.A., $\mathrm{N}^{\circ}$ 5, 273-290.

Vincent A., Malet P., 1977. Principes de fonctionnement intégré du blé. Le Tocsin du Radiateur, $\mathrm{N}^{\circ}$ 5, 282-288.

Wiegand C. L., Richardson A. J., 1984. Leaf area, light interception and yield estimates from spectral component analysis. Agron. J., 76, 543-548. 